\title{
The Influence of Preoperative Narcotic Consumption on Patient-Reported Outcomes of Lumbar Decompression
}

\author{
Conor P. Lynch, Elliot D. K. Cha, Shruthi Mohan, Cara E. Geoghegan, Caroline N. Jadczak, Kern Singh \\ Department of Orthopaedic Surgery, Rush University Medical Center, Chicago, IL, USA
}

\section{Study Design: Retrospective cohort.}

Purpose: This study aimed to assess the relationship between preoperative narcotic consumption and patient-reported outcomes (PRO) in patients undergoing minimally invasive (MIS) lumbar decompression (LD).

Overview of Literature: Previous studies report negative effects of narcotic consumption on perioperative outcomes and recovery; however, its impact on quality of life and surgical outcomes is not fully understood.

Methods: A surgical database was retrospectively reviewed for patients undergoing primary, single-level MIS LD from 2013 to 2020. Patients lacking preoperative narcotic consumption data were excluded. Demographics, spinal pathologies, and operative characteristics were collected. Patients were grouped based on preoperative narcotic consumption. Patient Health Questionnaire-9 (PHQ-9), Visual Analog Scale (VAS) for back and leg, Oswestry Disability Index (ODI), 12-item Short Form Physical Component Summary, and Patient-Reported Outcomes Measurement Information System physical function (PROMIS-PF) were collected preoperatively and postoperatively. Preestablished values were used to calculate achievement of minimum clinically important difference (MCID). Differences in mean PROs and MCID achievement between groups were evaluated.

Results: The cohort was 453 patients; 184 used preoperative narcotics and 269 did not. Significant differences were found in American Society of Anesthesiologists classification, ethnicity, insurance type, and estimated blood loss between groups. Significant differences were also found in preoperative PHQ-9, VAS leg, ODI, and PROMIS-PF between groups (all $p<0.05$ ). Mean postoperative PROs did not differ by group ( $p>0.05)$. A higher rate of MCID achievement was associated with the narcotic group for PHQ-9 and PROMIS-PF at 6 weeks (both $p \leq 0.050)$, VAS leg at 1 year $(p=0.009)$, and overall for ODI and PHQ-9 (both $p \leq 0.050)$.

Conclusions: Preoperative narcotic consumption was associated with worse preoperative depression, leg pain, disability, and physical function. In patients consuming preoperative narcotics, a higher proportion achieved an overall MCID for disability and depressive symptoms. Patients taking preoperative narcotic medications may report significantly worse preoperative PROs but demonstrate greater improvements in postoperative disability and mental health.

Keywords: Narcotics; Patient reported outcome measures; Patient health questionnaire; Minimal clinically important difference

Received Feb 17, 2021; Revised Mar 17, 2021; Accepted Mar 17, 2021

Corresponding author: Kern Singh

Department of Orthopaedic Surgery, Rush University Medical Center, 1611 W. Harrison St, Suite \#300, Chicago, IL, 60612, USA

Tel: +1-312-432-2373, Fax: +1-708-409-5179, E-mail: kern.singh@rushortho.com 


\section{Introduction}

With a lifetime prevalence of $49 \%-70 \%$, most people will experience low back pain at some point in their life [1]. When this pain becomes severe, narcotic medications are widely prescribed, leading to an exponential rise in use for pain management. In fact, approximately $40 \%$ of patients with newly diagnosed back or lower extremity pain were given an opiate prescription in the first year $[2,3]$. Surgical treatment may be indicated to alleviate symptoms when conservative measures fail to relieve intense pain caused by degeneration of the lumbar spine. Risks of narcotic dependence and addiction are well documented in lumbar spine surgery patients and can contribute to the substance abuse epidemic [4,5].

Minimally invasive (MIS) lumbar decompression (LD) is a common surgical treatment option for diseases that cause refractory low back pain such as lumbar spinal stenosis [6]. Previous lumbar spine studies have established preoperative narcotic use as a strong predictor of prolonged hospital stays and have also demonstrated that continuous postoperative narcotic use may lead to less favorable clinical outcomes [7,8]. In fact, Weiner et al. [9] and Hills et al. [10] reported that spine patients using narcotics preoperatively were less likely to experience a clinically significant improvement in postoperative pain and disability, as measured by Patient-Reported Outcomes Measurement Information System (PROMIS) pain interference and Oswestry Disability Index (ODI), respectively.

Chronic narcotic use has not only been demonstrated to affect physical health outcomes but has also been associated with poorer mental health status [11]; in fact, a study on lumbar and cervical spine patients reported that those who are preoperatively classified as depressed also had higher rates of narcotic use before surgery [12]. Notably, preoperative depression in LD patients has been found to hinder postoperative physical health improvement [13].

The potential for negative clinical and lifestyle outcomes in patients who continuously take narcotics is concerning for the field of spine surgery. Especially with LD being widely used as a highly effective procedure, a deeper understanding of the differences in preoperative characteristics of narcotic users and quantification of the postoperative implications of preoperative use may direct spine surgeons to more responsibly prescribe narcotics and mitigate postsurgical risks. To the best of our knowledge, no studies have comprehensively considered both physical and mental outcomes during the preoperative and postoperative periods among LD patients consuming narcotics. Therefore, this study aimed to assess the relationship between preoperative narcotic consumption and patientreported outcomes in patients undergoing MIS LD.

\section{Materials and Methods}

\section{Patient population}

This study was approved by the Institutional Review Board of Rush University Medical Center (ORA 14051301). Informed consents were obtained from patients before any study activity began. Study data were obtained via a retrospective review of a prospectively maintained surgical registry for lumbar spine procedures performed by a single attending surgeon at an academic institution from January 2013 to January 2020. The inclusion criteria were patients undergoing primary, elective, single-level MIS LD procedures for degenerative spinal pathology. Patients were excluded if information regarding preoperative narcotic consumption was incomplete, if operative duration or estimated blood loss (EBL) fell outside of 3 standard deviations from the mean, or if surgery was indicated because of infection, malignancy, or trauma.

\section{Data collection}

Demographic information for all patients such as age, gender, body mass index (BMI), smoking status, diabetes mellitus status, American Society of Anesthesiologists (ASA) classification, Charlson comorbidity index, ethnicity, and insurance/payment collected was recorded. Data regarding preoperative narcotic medication were collected by querying a state prescription monitoring program database. Preoperative narcotic use was defined as patients who received two or more narcotic prescriptions from our practice or had been prescribed narcotics by a provider outside of our practice. Narcotic medications included any prescription opioids in the preoperative period.

Perioperative information such as preexisting spinal pathology, operative duration (skin incision to closure, in minutes), EBL (in minutes), and postoperative length of stay (in hours) was recorded. Patient-reported outcome measures (PROM) were administered at preoperative and 6-week, 12-week, 6-month, 1-year, and 2-year postoperative time points. PROMs included Patient Health Ques- 
tionnaire-9 (PHQ-9) to evaluate depressive symptoms, Visual Analog Scale (VAS) to evaluate back and leg pain, ODI to evaluate disability, and the 12-item Short Form Physical Component Summary (SF-12 PCS) and PROMIS physical function $(\mathrm{PF})$ to evaluate $\mathrm{PF}$.

\section{Statistical analysis}

Stata MP ver. 16.1 (Stata Corp., College Station, TX, USA) was used for all calculations and statistical analysis.
Based on the described criteria for preoperative narcotic prescription, patients were grouped into narcotic and nonnarcotic groups. Chi-square analysis or Student $t$-test for categorical and continuous variables, respectively, was used to compare demographic and perioperative characteristics. Student $t$-test for independent samples was used to compare mean PROM scores between groups at each time point. Determinations of statistical significance may be unduly influenced by sample size and may not actually correlate to changes that are readily appreciable to

Table 1. Patient demographics

\begin{tabular}{|c|c|c|c|c|}
\hline Characteristic & Total $(n=453)$ & No preoperative narcotics ( $\mathrm{n}=269$ ) & Preoperative narcotics ( $\mathrm{n}=184$ ) & $p$-value ${ }^{a l}$ \\
\hline Age (yr) & $43.1 \pm 12.3$ & $42.8 \pm 12.4$ & $43.5 \pm 12.2$ & 0.548 \\
\hline Gender & & & & 0.573 \\
\hline Female & $31.1(141)$ & $30.1(81)$ & $32.6(60)$ & \\
\hline Male & $68.9(312)$ & $69.9(188)$ & $67.4(124)$ & \\
\hline Body mass index $\left(\mathrm{kg} / \mathrm{m}^{2}\right)$ & & & & 0.415 \\
\hline$<30$ & $62.0(281)$ & $63.6(171)$ & $59.8(110)$ & \\
\hline$\geq 30$ & $38.0(172)$ & $36.4(98)$ & $40.2(74)$ & \\
\hline Smoking status & & & & 0.760 \\
\hline Non-smoker & $84.3(382)$ & $84.8(228)$ & $83.7(154)$ & \\
\hline Smoker & $15.7(71)$ & $15.2(41)$ & $16.3(30)$ & \\
\hline Diabetes & & & & 0.521 \\
\hline Non-diabetic & $96.0(435)$ & $95.5(257)$ & $96.8(183)$ & \\
\hline Diabetic & $4.5(12)$ & $4.5(12)$ & $3.2(6)$ & \\
\hline ASA classification & & & & $<0.001$ \\
\hline$<2$ & $31.6(143)$ & $38.3(103)$ & $21.7(40)$ & \\
\hline$\geq 2$ & $68.4(310)$ & $61.7(166)$ & $78.3(144)$ & \\
\hline Charlson comorbidity index & & & & 0.531 \\
\hline$\leq 1$ & $77.5(331)$ & $78.6(198)$ & $76.0(133)$ & \\
\hline$>1$ & $22.5(96)$ & $21.4(54)$ & $23.8(43)$ & \\
\hline Ethnicity & & & & 0.035 \\
\hline White & $69.5(314)$ & $72.9(196)$ & $64.5(118)$ & \\
\hline African American & $12.0(54)$ & $9.3(25)$ & $15.9(29)$ & \\
\hline Hispanic & $11.7(53)$ & $9.7(26)$ & $14.8(27)$ & \\
\hline Asian & $4.2(19)$ & $4.5(12)$ & $3.8(7)$ & \\
\hline Other & $2.7(12)$ & $3.7(10)$ & $1.1(2)$ & \\
\hline Insurance & & & & $<0.001$ \\
\hline Medicare/Medicaid & $1.1(5)$ & $0.7(2)$ & $1.6(3)$ & \\
\hline Workers' compensation & $27.0(122)$ & $19.0(51)$ & $38.8(71)$ & \\
\hline Private & $71.9(325)$ & $80.3(216)$ & $59.6(109)$ & \\
\hline
\end{tabular}

Values are presented as mean \pm standard deviation or \% (number). Boldface indicates significance.

ASA, American Society of Anesthesiologists.

${ }^{a}$ Calculated using either chi-square test (for categorical variables) or a $t$-test (for continuous variables). 
patients [14]. Minimum clinically important difference (MCID) is defined as "a calculated threshold value in an outcome of interest that patients and clinicians perceive as clinically meaningful" [15]. To assess postoperative changes that are clinically significant and appreciable to the patient, rather than those that are purely of statistical significance, improvement in PROMs was quantified in terms of the MCID. Achievement of MCID after MIS LD was determined as a difference in PROM scores from preoperative baseline to postoperative follow-up that met or surpassed previously established thresholds for clinically significant improvement: PHQ-9=5.0 [16], VAS back=2.2 [17], VAS leg=5.0 [17], ODI=8.2 [17], SF-12 PCS=2.5 [17], and PROMIS-PF=4.5 [18]. To assess the relationship between narcotic consumption and patients' ability to realize meaningful improvement after surgery, a chi-square test was used to determine whether preoperative narcotic consumption was associated with the proportion of patients that achieved an MCID as a result of their MIS LD procedure at individual time points and overall (at any time point) for each PROM. A $p$-value of $\leq 0.05$ was considered statistically significant.

\section{Results}

Our overall study cohort included a total of 453 MIS LD patients, with a mean age of 43.1 years, a majority of whom were male (68.9\%) and nonobese (BMI of $<30 \mathrm{~kg} /$ $\left.\mathrm{m}^{2}\right)(62.0 \%)$ (Table 1). The cohort included 269 patients without preoperative narcotic prescriptions and 184 with preoperative narcotic prescriptions. Significant intergroup differences were observed in terms of ASA classification $(p<0.001)$, ethnicity $(p=0.035)$, and insurance collected $(p<0.001)$. The narcotic group had a greater proportion with ASA of $\geq 2$, more patients of African American and Hispanic ethnicity, and more patients receiving workers' compensation or Medicare/Medicaid.

The most common preoperative spinal pathology was recurrent herniated nucleus pulposus. Mean operative duration was 40.6 minutes, mean EBL was $28.0 \mathrm{~mL}$, and mean length of stay was 5.1 hours (Table 2). EBL was the only perioperative variable that differed significantly between nonnarcotic $(28.8 \mathrm{~mL})$ and narcotic $(26.8 \mathrm{~mL})$ groups ( $p=0.016$ ).

Preoperative PHQ-9 (5.4 versus 7.3, $p=0.012$ ), VAS leg ( 6.0 versus $6.6, p=0.032$ ), ODI ( 40.1 versus $45.0, p=0.010$ ), and PROMIS-PF (37.6 versus 34.8, $p=0.003$ ) scores were significantly worse for the narcotic group (Table 3 ). VAS back and SF-12 PCS did not significantly differ between groups preoperatively. No significant postoperative differences were observed between groups for any PROM at any time point (all $p>0.05$ ).

Most patients in both groups achieved an overall MCID in VAS back (58.5\% and 65.6\%), ODI (65.2\% and 76.5\%), SF-12 PCS (62.8\% and 62.7\%), and PROMIS-PF (61.9\% and 73.8\%). Rates of MCID achievement differed significantly between groups for PHQ-9 (21.9\% versus $37.4 \%$, $p=0.028)$ and PROMIS-PF ( $47.3 \%$ versus $67.9 \%, p=0.015$ ) at 6 weeks, VAS leg at 1 year $(25.0 \%$ versus $53.4 \%$, $p=0.009)$, and PHQ-9 (24.7\% versus $37.2 \%, p=0.044)$ and ODI ( $65.2 \%$ versus $76.5 \%, p=0.021)$ overall (Table 4$)$.

\section{Discussion}

Narcotic use is prevalent among patients with degenerative pathology of the lumbar spine and especially so among those who eventually undergo spinal surgery $[2,3]$. Although narcotic medications may provide short-

Table 2. Perioperative characteristics

\begin{tabular}{|c|c|c|c|c|}
\hline Variable & Total $(n=453)$ & No preoperative narcotics ( $n=269$ ) & Preoperative narcotics ( $\mathrm{n}=184$ ) & $p$-value ${ }^{\text {a) }}$ \\
\hline \multicolumn{5}{|l|}{ Spinal pathology } \\
\hline Recurrent herniated nucleus pulposus & $91.0(412)$ & $91.5(246)$ & $90.2(166)$ & 0.653 \\
\hline Central stenosis & $57.6(261)$ & $60.2(162)$ & $53.8(99)$ & 0.175 \\
\hline Foraminal stenosis & $44.4(201)$ & $46.5(125)$ & $41.3(76)$ & 0.277 \\
\hline Operative time (min) & $40.6 \pm 10.9$ & $40.3 \pm 10.8$ & $41.0 \pm 11.1$ & 0.545 \\
\hline Estimated blood loss (mL) & $28.0 \pm 8.6$ & $28.8 \pm 9.4$ & $26.8 \pm 7.1$ & 0.016 \\
\hline Length of stay (hr) & $5.1 \pm 6.8$ & $5.0 \pm 7.2$ & $5.1 \pm 6.1$ & 0.888 \\
\hline
\end{tabular}

Values are presented as \% (number) or mean \pm standard deviation. Boldface indicates significance.

${ }^{\text {a) }}$ Calculated using either chi-square test (for categorical variables) or $t$-test (for continuous variables). 
Table 3. Patient-reported outcomes between narcotic consumption groups

\begin{tabular}{|c|c|c|c|}
\hline Variable & $\begin{array}{c}\text { No preoperative } \\
\text { narcotics }\end{array}$ & $\begin{array}{c}\text { Preoperative } \\
\text { narcotics }\end{array}$ & $p$-value ${ }^{\text {a) }}$ \\
\hline \multicolumn{4}{|l|}{ PHO-9 } \\
\hline Preoperative & $5.4 \pm 4.8(142)$ & $7.3 \pm 6.5(86)$ & 0.012 \\
\hline 6 weeks & $3.7 \pm 4.3(113)$ & $5.0 \pm 5.2(72)$ & 0.705 \\
\hline 12 weeks & $4.5 \pm 6.7(61)$ & $4.5 \pm 6.2(48)$ & 0.998 \\
\hline 6 months & $4.8 \pm 5.9(52)$ & $4.2 \pm 5.6$ (39) & 0.618 \\
\hline 1 year & $3.2 \pm 4.6(48)$ & $4.6 \pm 6.5(31)$ & 0.245 \\
\hline 2 years & $5.5 \pm 6.2(18)$ & $5.2 \pm 6.7(17)$ & 0.904 \\
\hline \multicolumn{4}{|l|}{ VAS back } \\
\hline Preoperative & $6.0 \pm 2.6(241)$ & $6.2 \pm 2.4(163)$ & 0.387 \\
\hline 6 weeks & $2.8 \pm 2.6(202)$ & $3.1 \pm 2.7(154)$ & 0.240 \\
\hline 12 weeks & $3.0 \pm 2.8(112)$ & $3.1 \pm 2.6(90)$ & 0.772 \\
\hline 6 months & $3.6 \pm 3.0(69)$ & $3.1 \pm 2.8(59)$ & 0.349 \\
\hline 1 year & $3.4 \pm 3.0(49)$ & $2.8 \pm 2.6(46)$ & 0.358 \\
\hline 2 years & $4.0 \pm 3.0(49)$ & $4.4 \pm 2.6(15)$ & 0.705 \\
\hline \multicolumn{4}{|l|}{ VAS leg } \\
\hline Preoperative & $6.0 \pm 2.5(197)$ & $6.6 \pm 2.6(147)$ & 0.032 \\
\hline 6 weeks & $2.8 \pm 2.7(156)$ & $3.0 \pm 3.0$ (138) & 0.488 \\
\hline 12 weeks & $2.9 \pm 2.9(92)$ & $2.8 \pm 2.8(81)$ & 0.849 \\
\hline 6 months & $310 \pm 2.9(62)$ & $2.6 \pm 2.7(57)$ & 0.399 \\
\hline 1 year & $2.7 \pm 2.6(49)$ & $2.3 \pm 2.9(46)$ & 0.479 \\
\hline 2 years & $3.4 \pm 2.8(27)$ & $2.7 \pm 3.4(15)$ & 0.503 \\
\hline \multicolumn{4}{|l|}{ ODI } \\
\hline Preoperative & $40.1 \pm 17.2(207)$ & $45.0 \pm 18.1(153)$ & 0.010 \\
\hline 6 weeks & $24.6 \pm 18.6(170)$ & $26.8 \pm 18.7(146)$ & 0.305 \\
\hline 12 weeks & $24.7 \pm 21.1(104)$ & $24.5 \pm 20.1$ (87) & 0.966 \\
\hline 6 months & $28.4 \pm 22.2(68)$ & $25.2 \pm 20.9(60)$ & 0.399 \\
\hline 1 year & $22.4 \pm 18.6(54)$ & $23.0 \pm 21.5(47)$ & 0.870 \\
\hline 2 years & $25.4 \pm 18.2(27)$ & $25.9 \pm 21.4(15)$ & 0.942 \\
\hline \multicolumn{4}{|l|}{ SF-12 PCS } \\
\hline Preoperative & $32.2 \pm 8.0$ (191) & $32.0 \pm 8.5(142)$ & 0.880 \\
\hline 6 weeks & $38.0 \pm 10.2(135)$ & $38.0 \pm 10.6(102)$ & 0.981 \\
\hline 12 weeks & $41.2 \pm 11.2(77)$ & $43.2 \pm 10.8(60)$ & 0.303 \\
\hline 6 months & $41.2 \pm 10.9(63)$ & $43.0 \pm 11.5(55)$ & 0.391 \\
\hline 1 year & $42.3 \pm 10.6(66)$ & $43.0 \pm 11.4(52)$ & 0.709 \\
\hline 2 years & $43.1 \pm 10.6(33)$ & $43.3 \pm 11.4(32)$ & 0.933 \\
\hline \multicolumn{4}{|l|}{ PROMIS-PF } \\
\hline Preoperative & $37.6 \pm 6.4(134)$ & $34.8 \pm 7.3(84)$ & 0.003 \\
\hline 6 weeks & $43.3 \pm 8.0(100)$ & $43.4 \pm 8.8(64)$ & 0.932 \\
\hline 12 weeks & $47.2 \pm 10.3(61)$ & $46.6 \pm 9.8(45)$ & 0.761 \\
\hline 6 months & $43.7 \pm 9.4(52)$ & $44.8 \pm 9.2(39)$ & 0.569 \\
\hline 1 year & $46.2 \pm 10.9(48)$ & $46.4 \pm 9.7$ (32) & 0.961 \\
\hline 2 years & $45.1 \pm 7.2(32)$ & $48.2 \pm 10.8(21)$ & 0.217 \\
\hline
\end{tabular}

Values are presented as mean \pm standard deviation (number of patients). Boldface indicates significance.

PHQ-9, Patient Health Questionnaire-9; VAS, Visual Analog Scale; ODI, Oswestry Disability Index; SF-12 PCS, 12-item Short Form Physical Component Summary; PROMIS-PF, Patient-Reported Outcomes Measurement Information System physical function.

${ }^{a}$ Calculated using $t$-test to compare intergroup differences.
Table 4. Achievement of minimum clinically important difference

\begin{tabular}{|c|c|c|c|}
\hline Variable & $\begin{array}{l}\text { No preoperative } \\
\text { narcotics }\end{array}$ & $\begin{array}{l}\text { Preoperative } \\
\text { narcotics }\end{array}$ & $p$-value ${ }^{\text {al }}$ \\
\hline \multicolumn{4}{|l|}{ PHO-9 } \\
\hline Overall & $24.7(35)$ & $37.2(32)$ & 0.044 \\
\hline 6 weeks & $21.9(23)$ & $37.4(26)$ & 0.028 \\
\hline 12 weeks & $26.8(15)$ & $37.2(16)$ & 0.268 \\
\hline 6 months & $24.4(11)$ & $30.3(11)$ & 0.564 \\
\hline 1 year & $9.8(4)$ & $4.2(1)$ & 0.414 \\
\hline 2 years & $12.5(2)$ & $7.7(1)$ & 0.672 \\
\hline \multicolumn{4}{|l|}{ VAS back } \\
\hline Overall & $58.5(141)$ & $65.6(107)$ & 0.148 \\
\hline 6 weeks & $60.6(114)$ & $58.7(82)$ & 0.706 \\
\hline 12 weeks & $52.4(54)$ & $61.3(49)$ & 0.233 \\
\hline 6 months & $54.7(35)$ & $61.1(33)$ & 0.482 \\
\hline 1 year & $50.0(22)$ & $60.5(26)$ & 0.326 \\
\hline 2 years & $38.5(10)$ & $50.0(7)$ & 0.481 \\
\hline \multicolumn{4}{|l|}{ VAS leg } \\
\hline Overall & $37.6(74)$ & $43.5(64)$ & 0.263 \\
\hline 6 weeks & 35.6 (52) & 33.6 (42) & 0.728 \\
\hline 12 weeks & $37.2(32)$ & $35.1(25)$ & 0.796 \\
\hline 6 months & $33.3(19)$ & $45.1(23)$ & 0.211 \\
\hline 1 year & $25.0(11)$ & $53.4(22)$ & 0.009 \\
\hline 2 years & $31.8(7)$ & $41.7(5)$ & 0.566 \\
\hline \multicolumn{4}{|l|}{ ODI } \\
\hline Overall & 65.2 (135) & $76.5(117)$ & 0.021 \\
\hline 6 weeks & $64.3(99)$ & $70.5(91)$ & 0.264 \\
\hline 12 weeks & $69.2(63)$ & 78.4 (58) & 0.186 \\
\hline 6 months & $61.0(36)$ & $75.9(41)$ & 0.089 \\
\hline 1 year & $66.7(30)$ & 76.2 (32) & 0.327 \\
\hline 2 years & $52.2(12)$ & $76.9(10)$ & 0.143 \\
\hline \multicolumn{4}{|l|}{ SF-12 PCS } \\
\hline Overall & $62.8(120)$ & $62.7(89)$ & 0.977 \\
\hline 6 weeks & $60.7(71)$ & $59.3(54)$ & 0.844 \\
\hline 12 weeks & $68.2(45)$ & $69.2(36)$ & 0.903 \\
\hline 6 months & $64.9(37)$ & $63.8(30)$ & 0.909 \\
\hline 1 year & $79.0(49)$ & 73.9 (34) & 0.533 \\
\hline 2 years & $78.1(25)$ & $75.9(22)$ & 0.834 \\
\hline \multicolumn{4}{|l|}{ PROMIS-PF } \\
\hline Overall & $61.9(83)$ & 73.8 (62) & 0.071 \\
\hline 6 weeks & $47.3(43)$ & $67.9(38)$ & 0.015 \\
\hline 12 weeks & $65.5(36)$ & $81.1(30)$ & 0.103 \\
\hline 6 months & $56.8(25)$ & $71.0(22)$ & 0.212 \\
\hline 1 year & $61.5(24)$ & $74.1(20)$ & 0.288 \\
\hline 2 years & $67.9(19)$ & $82.4(14)$ & 0.286 \\
\hline
\end{tabular}

Values are presented as \% (number of patients). Boldface indicates significance.

PHQ-9, Patient Health Questionnaire-9; VAS, Visual Analog Scale; ODI, Oswestry Disability Index; SF-12 PCS, 12-item Short Form Physical Component Summary; PROMIS-PF, Patient-Reported Outcomes Measurement Information System physical function.

${ }^{\text {al }}$ Calculated using chi-square to compare intergroup differences. 
term relief for some patients, their use is associated with a host of physical, psychological, and socioeconomic issues $[4,5]$. Our cohort demonstrated significant preoperative differences on the basis of narcotic use in terms of demographics and physical and mental health. Postoperatively, patients who had previously used narcotic medications did not significantly differ in mean PROM scores and demonstrated greater rates of meaningful improvement in disability and depressive symptoms.

Preoperative narcotic use was associated with several important demographic differences in our cohort. A significantly larger proportion of patients in the narcotic group were receiving workers' compensation. Narcotic use has been well studied in the workers' compensation population, particularly with regard to outcomes of spine surgery. In their study of worker's compensation in patients undergoing lumbar discectomy, O'Donnell et al. [19] demonstrated that even short-term preoperative opioid use was associated with significantly lower rates of return to work and greater overall medical cost. Although a majority of both groups were of White ethnicity, the narcotic group had a significantly greater distribution of African American and Hispanic patients. Previous studies provide evidence for similar racial differences in narcotic use among African Americans [20]. However, results differed with regard to Hispanic patients, for whom King and Liu [20] reported lower overall rates of opioid prescriptions. Moreover, our study observed a significantly greater proportion of patients with an ASA classification of 2 or greater in the narcotic group. Intended as a general measure of health and medical risk, this difference suggests patients taking narcotics preoperatively may tend to be in poorer health overall [21].

In line with our finding regarding ASA classification, preoperatively, the narcotic group was significantly worse off in terms of their physical health, demonstrating more severe leg pain, disability, and PF deficit before undergoing MIS LD. Unsurprisingly, greater preoperative pain has been reported to predict narcotic use in patients undergoing spine surgery [12]. Similarly, Mesfin et al. [22] demonstrated significantly more severe pain and disability in patients who consumed narcotics before spine surgery.

In addition to differences in physical well-being, the narcotic group also demonstrated significantly higher depressive symptom severity at the preoperative time point. In their study of 583 patients undergoing spine surgery, Armaghani et al. [12] found that preoperative depression, as measured by the Zung Depression Scale (ZDS), was significantly associated with preoperative narcotic use. Furthermore, Armaghani et al. [12] found that patients who were depressed $(p<0.001)$ and patients who were anxious $(p<0.001)$ had significantly higher preoperative morphine equivalent dosage than those who were not. Interestingly, these authors established a direct correlation between ZDS score and preoperative morphine equivalents $(p<0.000001)$. Although Virk et al. [23] measured depression through PROMIS mental health scores, they too found that preoperative morphine equivalent dosage was correlated with poorer mental health.

Previous findings regarding postoperative outcomes related to preoperative narcotic consumption have been somewhat more mixed. Lee et al. [24] reported that preoperative opioid use was associated with worse outcomes in SF-12, EuroQoL 5-dimension, and ODI at both 3 months and 1 year, even when controlling for preoperative depression. Likewise, Weiner et al. [9] reported a significant difference in improvement of PROMIS pain interference scores based on narcotic consumption and found that most patients who reported opioid use before surgery did not achieve an MCID. Conversely, Virk et al. [23] did not observe a significant difference in outcomes on the basis of preoperative narcotic use. In their study of 126 microdiscectomy patients, Virk et al. [23] found no significant differences in terms of MCID achievement for ODI, PROMIS physical health scores, or mental health scores at 4 weeks, 6 months, or 1 year postoperatively. Results of our analysis regarding PF are most in line with those of Virk et al. [23].

In terms of mean PROM scores, no significant postoperative differences were found between the narcotic and nonnarcotic groups. Interestingly, the only significant differences in MCID achievement were actually in favor of the narcotic group. In particular, significant overall differences were found in MCID achievement for ODI, short-term differences for PROMIS-PF, and long-term differences in VAS leg. Our results regarding disability improvement may seem somewhat surprising given the negative outcomes related to narcotic consumption reported by previous studies. However, although mean postoperative PROM scores were similar for both groups in our study, the narcotic group had significantly more severe symptoms in almost all categories preoperatively. Therefore, it is possible that the greater rate of MCID achievement in the narcotic group could be explained by 
a greater degree of "room" for improvement in these patients.

Although associations of narcotic use with physical health outcomes have been relatively well published, literature regarding postoperative mental health outcomes related to preoperative narcotic use is limited. Lee et al. [24] demonstrated that preoperative opioid use was associated with quality of life at both 3-month and 1-year postoperative follow-ups. Virk et al. [23] is one of the few studies that specifically examined postoperative mental health outcomes related to preoperative narcotic use. Their results indicated that although preoperative opioid use was correlated with PROMIS mental health scores at 3 months postoperatively, there was no difference in achievement of MCID between opioid-using and opioid-naive groups after microdiscectomy [23]. In contrast to the results of Virk et al. [23], our analysis did reveal a significant difference in meaningful improvement in depressive symptoms based on preoperative narcotic use. Although overall rates of MCID achievement for PHQ-9 were generally low, a greater proportion of the narcotic group reached a meaningful difference in this mental health outcome than in the nonnarcotic group. As with our findings regarding disability, this result was not one we had expected. However, given the more severe depressive symptoms reported by the narcotic group, it similarly may be explained by a greater potential for improvement. However, our results regarding achievement of a meaningful improvement in depressive symptoms come with a significant caveat. Although other MCID values used in this study were derived from analyses specific to spine surgery, no such calculation has been published for PHQ-9. Our PHQ-9 MCID value was derived from a study of responsiveness to treatments specifically targeted at depression [16]. Relatively low rates of MCID achievement for PHQ-9 in our study may be partly explained by the fact that MIS LD is aimed at correcting physical pathology, with improvements in depressive symptoms being a secondary (but not unimportant) outcome. Therefore, a meaningful difference obtained after specific psychological intervention may be an unfair comparison for improvement in depressive symptoms after spine surgery.

Although prolonged use of narcotic medications is associated with a host of well-documented physical and psychosocial risks, our analysis may assuage some concerns of physicians considering spine surgery for patients who have been prescribed narcotics preoperatively. This information may be helpful both when considering risks vs benefits of preoperative narcotic prescriptions and in preoperative counseling in terms of what patients may be able to expect after LD surgery. In particular, our finding regarding PHQ-9 improvement in the narcotic cohort highlights a key domain in which these patients may improve, and efforts should be made to maximize these benefits of decompression surgery.

There are several limitations in our study. First, our data are based on procedures performed by one attending surgeon at a single academic institution, which may limit generalizability of our findings to other populations. Second, the self-reported nature of our outcomes data may introduce an element of bias to our results. Third, we were not able to include detailed information about longterm postoperative narcotic use. We were able to query our state's prescription monitoring program to obtain information regarding preoperative narcotic prescriptions. Nearly all patients are prescribed some amount of narcotic medications postoperatively, but this information would not allow us to accurately quantify the amount of medication patients actually consumed and self-reported narcotic data may be unreliable. Finally, gabapentinoids have been used for the management of spinal pain, but were not prescribed preoperatively in our cohort. Future studies should explore the implications of these medications for postoperative outcomes.

\section{Conclusions}

Preoperative prescription for narcotic mediation was associated with significantly more severe depression, leg pain, disability, and PF deficits before surgery. However, these differences became significantly less pronounced after MIS LD. In fact, a greater proportion of patients who consumed narcotics preoperatively achieved an MCID in disability and depressive symptoms than those who had not used narcotics before surgery. Our results suggest that preoperative narcotic consumption may not interfere with a patient's ability to achieve an MCID after MIS LD and highlight an increased potential for improvement in initially poor mental health in this population.

\section{Conflict of Interest}

No potential conflict of interest relevant to this article was reported. 


\section{Author Contributions}

Conception and design: CPL, EDKC, KS; data acquisition: KS; analysis of data: CPL, EDKC; drafting manuscript: CPL, EDKC, SM; administrative support: SM, CEG, CNJ; critical revision: CPL, EDKC, SM, CEG, CNJ, KS; and supervision: KS.

\section{References}

1. Koes BW, van Tulder MW, Thomas S. Diagnosis and treatment of low back pain. BMJ 2006;332:1430-4.

2. Hilliard PE, Waljee J, Moser S, et al. Prevalence of preoperative opioid use and characteristics associated with opioid use among patients presenting for surgery. JAMA Surg 2018;153:929-37.

3. Azad TD, Vail D, Bentley J, et al. Initial provider specialty is associated with long-term opiate use in patients with newly diagnosed low back and lower extremity pain. Spine (Phila Pa 1976) 2019;44:211-8.

4. Esfahani K, Naik BI, Dunn LK. Chronic opioid use after spine surgery: what is the prescription for reducing opioid dependence? J Spine Surg 2018;4:8179.

5. Centers for Disease Control and Prevention. Understanding the epidemic [Internet]. Atlanta (GA): Centers for Disease Control and Prevention; 2020 [cited 2020 Oct 6]. Available from: https://www.cdc.gov/drugoverdose/epidemic/index.html.

6. Kleinstueck FS, Fekete T, Jeszenszky D, et al. The outcome of decompression surgery for lumbar herniated disc is influenced by the level of concomitant preoperative low back pain. Eur Spine J 2011;20:1166-73.

7. Deyo RA, Hallvik SE, Hildebran C, et al. Use of prescription opioids before and after an operation for chronic pain (lumbar fusion surgery). Pain 2018;159:1147-54.

8. Jain N, Phillips FM, Weaver T, Khan SN. Preoperative chronic opioid therapy: a risk factor for complications, readmission, continued opioid use and increased costs after one- and two-level posterior lumbar fusion. Spine (Phila Pa 1976) 2018;43:1331-8.

9. Weiner JA, Snavely JE, Johnson DJ, Hsu WK, Patel AA. Impact of preoperative opioid use on postoperative patient-reported outcomes in lumbar spine surgery patients. Clin Spine Surg 2021;34:E154-9.

10. Hills JM, Pennings JS, Archer KR, et al. Preoperative opioids and 1-year patient-reported outcomes after spine surgery. Spine (Phila Pa 1976) 2019;44:887-95.

11. Jensen MK, Thomsen AB, Hojsted J. 10-Year followup of chronic non-malignant pain patients: opioid use, health related quality of life and health care utilization. Eur J Pain 2006;10:423-33.

12. Armaghani SJ, Lee DS, Bible JE, et al. Preoperative narcotic use and its relation to depression and anxiety in patients undergoing spine surgery. Spine (Phila Pa 1976) 2013;38:2196-200.

13. Sinikallio S, Aalto T, Airaksinen O, et al. Depression is associated with poorer outcome of lumbar spinal stenosis surgery. Eur Spine J 2007;16:905-12.

14. Copay AG, Subach BR, Glassman SD, Polly DW Jr, Schuler TC. Understanding the minimum clinically important difference: a review of concepts and methods. Spine J 2007;7:541-6.

15. Chung AS, Copay AG, Olmscheid N, Campbell D, Walker JB, Chutkan N. Minimum clinically important difference: current trends in the spine literature. Spine (Phila Pa 1976) 2017;42:1096-105.

16. Lowe B, Unutzer J, Callahan CM, Perkins AJ, Kroenke K. Monitoring depression treatment outcomes with the patient health questionnaire-9. Med Care 2004;42:1194-201.

17. Parker SL, Mendenhall SK, Shau DN, et al. Minimum clinically important difference in pain, disability, and quality of life after neural decompression and fusion for same-level recurrent lumbar stenosis: understanding clinical versus statistical significance. J Neurosurg Spine 2012;16:471-8.

18. Steinhaus ME, Iyer S, Lovecchio F, et al. Minimal clinically important difference and substantial clinical benefit using PROMIS CAT in cervical spine surgery. Clin Spine Surg 2019;32:392-7.

19. O’Donnell JA, Anderson JT, Haas AR, et al. Preoperative opioid use is a predictor of poor return to work in workers' compensation patients after lumbar diskectomy. Spine (Phila Pa 1976) 2018;43:594-602.

20. King C, Liu X. Racial and ethnic disparities in opioid use among US adults with back pain. Spine (Phila $\mathrm{Pa}$ 1976) 2020;45:1062-6.

21. American Society of Anesthesiologists. ASA Physical Status Classification System [Internet]. Schaumburg (IL): American Society of Anesthesiologists; 2014 [cited 2020 Oct 10]. Available from: https://www.asahq.org/standards-and-guidelines/ asa-physical-status-classification-system. 
22. Mesfin A, Lenke LG, Bridwell KH, et al. Does preoperative narcotic use adversely affect outcomes and complications after spinal deformity surgery?: a comparison of nonnarcotic-with narcotic-using groups. Spine J 2014;14:2819-25.

23. Virk S, Sandhu M, Qureshi S, Albert T, Sandhu H. How does preoperative opioid use impact postoperative health-related quality of life scores for pa- tients undergoing lumbar microdiscectomy? Spine J 2020;20:1196-202.

24. Lee D, Armaghani S, Archer KR, et al. Preoperative opioid use as a predictor of adverse postoperative self-reported outcomes in patients undergoing spine surgery. J Bone Joint Surg Am 2014;96:e89. 